\title{
Le traducteur juridique algérien face à la difficulté de traduire certains termes juridiques arabes
}

\author{
Nourredine BESSADI \\ Université Mouloud Mammeri \\ Université Abderrahmane Mira de Béjaïa
}

En réponse à la question de savoir quels problèmes spécifiques (sociolinguistiques, linguistiques et culturels) pose la traduction d'autres genres que la littérature francophone, nous avons choisi de parler des difficultés inhérentes à la traduction des textes à teneur juridique en Algérie, plus particulièrement des problèmes rencontrés dans le passage de l'arabe au français lors de la traduction de tels textes.

L'intérêt porté à ce genre de traduction est venu de notre travail sur des textes de loi algériens rédigés et diffusés en deux langues, l'arabe et le français. En Algérie, et sur le plan linguistique, on constate en effet un bilinguisme de fait en matière juridique. Dès le lendemain de l'indépendance, en 1962, l'État algérien a puisé dans le droit musulman et la langue arabe les attributs d'une identité algérienne à recouvrer tout en puisant dans le droit français et, ce faisant, dans la langue française le stock de notions et de modes de raisonnements susceptibles de lui donner accès à la modernité à laquelle il prétendait.

Ainsi, travaillant sur un corpus écrit, nous nous sommes retrouvé en face de textes rédigés en deux langues : l'arabe - l'arabe classique s'entend - et le français. Fait hauteent paradoxal, ce même droit diffusé en deux langues pose la langue arabe comme seule langue officielle ${ }^{1}$. Par ailleurs, la voix officielle n'omet pas de préciser que la version arabe est la version source et que la version française n'en est que la traduction. Sur la version française du Journal Officiel de la République algérienne par exemple, on peut lire la mention «Traduction de l'arabe ». Une mention qui laisse toutefois perplexe nombre de spécialistes du droit algérien; eux pour qui c'est la version française qui continue d'être la version de référence ${ }^{2}$.

Néanmoins, notre propos n'étant pas ici de vérifier la fiabilité de telle ou telle autre thèse, nous nous attacherons à parler du caractère spécifique de ce genre de traduction particulier qu'est la traduction juridique, puis nous soulèverons les obstacles que rencontre le traducteur des textes à teneur juridique en Algérie lors du passage de l'arabe au français, et ce, à la lumière de la traduction du Code de la famille.

\section{Le caractère spécifique de la traduction juridique}

Tout d'abord, il faut souligner que comme toute traduction, celle des textes juridiques suppose l'intégration de connaissances à la fois linguistiques et extralinguistiques. Elle est ainsi considérée comme étant une traduction technique et culturelle, scientifique et sociale.

Sa technicité consiste en ses outils spécialisés, son caractère culturel repose sur le fait qu'elle se réfère constamment à des institutions humaines différentes d'un pays à l'autre. Son aspect scientifique se

\footnotetext{
${ }^{1}$ L'Etat algérien a en effet adopté une politique linguistique volontariste dite politique d'arabisation, et le secteur juridique a été le secteur inaugural de cette politique.

${ }^{2} \mathrm{Il}$ y a tout de même la place à une troisième possibilité, celle de la corédaction. Les arabisants rédigeraient de leur côté la version en arabe et les francisants s'occuperaient de la version en français, les uns ne traduisant pas à proprement parler le travail des autres.
} 
concrétise par sa méthode rigoureuse, sa dimension sociale se reflète dans son adaptation continue et son évolution dynamique. (Koustivitis 226)

Cependant, alors qu'une traduction purement technique (la traduction médicale par exemple) se caractérise par l'unité du signifié-source et du signifié-cible, la traduction juridique, quant à elle, est confrontée à une difficulté de taille : infléchir le signifié pour en rendre compte dans une langue juridique des institutions et, partant, pour rendre compte de notions autres que celles pour lesquelles cette langue a été conçue au départ. De la différence des systèmes et des institutions juridiques résulte l'absence de relations biunivoques, que ce soit au niveau terminologique, phraséologique, et au niveau du discours tout entier.

Lorsqu'il s'agit de traduire un texte, le traducteur est amené à opérer un transfert de son contenu culturel. Le texte juridique, porteur de notions chargées d'histoire et de traditions, de par sa charge culturelle, est un très bon exemple des difficultés relatives à ce genre de transfert. En effet, chaque langue imprimant de son sceau le système juridique auquel elle donne vie et de l'évolution de laquelle elle participe, la question qui se pose est la suivante : le texte d'arrivée serat-il satisfaisant dans la lettre (équivalence linguistique) et dans l'esprit (portée juridique) pour un destinataire d'une culture différente de celle du texte de départ ? Il est clair que le droit s'exprime de façons différentes au sein de la même langue et du même système juridique et que cette différence est accentuée lors du passage d'un système ou d'une langue à l'autre par le biais de la traduction.

L'opération traduisante exige la maîtrise de la technique de la traduction qui mobilise des compétences particulières auxquelles ne peut répondre le simple fait de maitriser une ou plusieurs langues étrangères. Il est à ce propos très pertinent de rappeler que le traducteur juridique, comme tout autre traducteur d'ailleurs, ne travaille pas sur les langues, mais sur des discours. La parfaite connaissance de la langue est certes essentielle pour qui veut s'adonner à l'art de la traduction. Néanmoins, ce dernier doit non seulement être familiarisé avec les différentes formes du discours juridique, avec ses subtilités terminologiques et syntaxiques, et connaître l'arrière-plan socio-culturel qui a vu naitre le texte juridique qui constitue sa matière, mais aussi être préparé et rompu aux techniques de la traduction susceptibles d'être mobilisées dans un domaine aussi délicat que celui du droit.

L'influence du système juridique sur la langue apparaît clairement à la lumière de la traduction juridique. On distingue deux situations différentes :

- La traduction peut s'effectuer sur la base d'un seul et même système juridique mais dans plusieurs langues. C'est le cas des pays plurilingues qui connaissent un seul système juridique tels que la Belgique et la Suisse.

- La traduction peut également s'effectuer à la base de plusieurs systèmes juridiques et dans plusieurs langues. C'est le cas, par exemple, des pays plurilingues qui connaissent un «bijuridisme» (Gémar 1995 : 151) tels que le Canada ${ }^{3}$. C'est le cas aussi de l'Algérie où le droit français croise le droit musulman et où le français côtoie l'arabe ${ }^{4}$.

Nul doute qu'il est plus simple de traduire un seul droit en plusieurs langues que de traduire deux droits distincts en plusieurs langues. C'est d'ailleurs au premier cas de figure qu'Emmanuel Didier réserve le nom de traduction juridique qui, selon lui, peut être pratiquée par un non-juriste. Le deuxième est appelé, toujours selon ses termes, transposition juridique.

\footnotetext{
${ }^{3} \mathrm{Au}$ Canada, ce bijuridisme concerne plus particulièrement les lois fédérales.

${ }^{4}$ Ce bilinguisme est qualifié de « honteux » par Ramdane Babadji puisque la seule langue reconnue par l'Etat algérien est la langue arabe. (Babadji, Ramdane. « Désarroi bilingue : note sur le bilinguisme juridique en Algérie ». Droit et Société, 15, 1990, p. 207-217)
} 
Il ne faut pas confondre la traduction juridique et la transposition juridique ${ }^{5}$. La traduction juridique est l'opération de transfert d'un message juridique, dans un seul système juridique, d'une langue vers une autre langue. La transposition juridique est l'opération de transfert d'un message juridique émis dans une langue et dans un système juridique, vers une autre langue et un autre système juridique. (9)

En effet, il ne sera pas question dans la première situation d'avoir recours au droit comparé puisque l'on se place dans le même système juridique. Les difficultés de traduction se poseront donc essentiellement au niveau de la forme linguistique, c'est-à-dire au niveau grammatical, syntaxique et stylistique.

Dans la deuxième situation, par contre, et c'est le cas pour la traduction juridique en Algérie, le recours au droit comparé s'avère indispensable et les difficultés de traduction seront doubles : celles se rapportant à la forme linguistique, inhérentes à tout type de traduction, et celles essentiellement liées au contenu juridique et aux problèmes propres au transfert entre cultures juridiques distinctes.

\section{Les difficultés inhérentes à la traduction du droit en Algérie}

Dans le cas algérien on est en face de deux cultures juridiques, la culture juridique civiliste et la culture juridique musulmane, issues de deux langues dont les systèmes linguistiques sont très différents: le français, une langue indo-européenne dont les descriptions grammaticales s'inscrivent dans la tradition occidentale, profondément marquée par la philosophie aristotélicienne, et l'arabe qui est une langue sémitique dont la tradition grammaticale reflète une pensée linguistique largement déterminée par l’interprétation du texte sacré qu'est le Coran.

Nous avons choisi d'illustrer notre propos par des exemples tirés du Code de la famille ${ }^{6}$ tant ce texte est très représentatif des difficultés se rapportant au transfert de l'une des deux langues vers l'autre dans le domaine juridique.

D'emblée, il est à noter que l'en-tête en caractères gras «RÉPUBLIQUE ALGÉRIENNE DÉMOCRATIQUE ET POPULAIRE» suivi de «MINISTĖRE DE LA JUSTICE », ainsi que l'intitulé du texte "CODE DE LA FAMILLE », sont curieusement absents dans l'énoncé en langue arabe. Cela est-il à mettre à l'actif de ceux qui soutiennent que c'est le texte en langue française qui demeure la version source? La tentation est grande même si le mot «bis», en petits caractères, accompagne systématiquement les numéros des pages du texte en français.

Ainsi, le premier chapitre du livre premier a pour titre en langue française «Des fiançailles «El Khitba » et du mariage ». Le titre correspondant dans l'énoncé en langue arabe est «El Khitba wa Z'rawadj». Celui-ci, si on le rend littéralement en français, donnera «Les fiançailles et le mariage ». Il apparait donc clairement qu'il y a redondance dans l'énoncé en langue française car les mots fiançailles et El Khitba se suivent alors qu'ils renvoient, a priori, à la même réalité. S'agit-il donc d'une erreur d'ordre typographique dans la mesure où, pour mieux le mettre en évidence, il aurait pu y avoir mise à la fois entre parenthèses et en italique à la place d'une mise entre guillemets, le mot étant étranger à la langue de traduction? Un autre exemple, de même nature, semble confirmer cette hypothèse. L'article 15 (Ordonnance $\mathrm{n}^{\circ}$ 05-02 du 27 février 2005) stipule dans le texte en langue française que : «La dot est fixée dans le contrat de mariage, que

\footnotetext{
${ }^{5}$ C'est l'auteur qui souligne.

${ }^{6} \mathrm{Il}$ s'agit plus exactement de la 4e édition, dépôt légal 406-2003.

${ }^{7}$ La mise entre guillemets du mot El Khitba est du traducteur-rédacteur.
} 
son versement soit immédiat ou à terme. À défaut de la fixation du montant de la dot, la dot de parité « sadaq el mithl » est versée à l'épouse. » Il y a encore redondance puisque Sadaq el mithl est le mot arabe correspondant à dot de parité.

Par ailleurs, la mise entre parenthèses est utilisée dans d'autres cas, pourtant semblables. Ainsi des sous-titres du deuxième chapitre « De la retraite légale (Idda) » et «Du droit de garde (Hadana)». Les mots Idda et Hadana étant les termes arabes correspondant respectivement à retraite légale et droit de garde, leur mise entre parenthèses est naturellement justifiée.

Cette confusion entre mise entre guillemets et mise entre parenthèses est très manifeste dans l'article 46 qui stipule que «L'adoption (Tabanni) est interdite par la (chari'â) et la loi ». En effet, si la première mise entre parenthèses est justifiée - Tabanni étant le mot arabe correspondant au mot français adoption - la deuxième ne l'est pas puisque le mot chari'â, qui est d'ailleurs actuellement passé dans la langue française sous la forme charia, n'est pas précédé d'un autre mot français de même sens. Une mise entre guillemets aurait donc été plus appropriée.

À cet égard, le cas des sous-titres du neuvième chapitre est encore plus illustratif. On peut en effet lire :

- Le cas dit (al aqdariya et al ghora);

- Le cas dit (almuchtaraka);

- Le cas dit (al gharawayn);

- Le cas dit (al mubahala);

- Le cas dit (al minbariya);

Les mots mis entre parenthèses, qui sont des mots arabes, ne sont pourtant précédés d'aucun mot français correspondant. Leur mise entre parenthèses est clairement abusive. Là, encore plus que dans le cas du mot chari'â, une mise entre guillemets aurait été largement plus justifiée.

Prenons maintenant l'exemple du terme juridique arabe wali, inséré sous cette forme (transcription phonétique) à certains endroits du texte en langue française alors qu'il est rendu par le mot tuteur dans d'autres endroits du même texte. On peut lire notamment à la fin de l'article 11 : «Le juge est le tuteur de la personne qui en est dépourvue. » Il est intéressant à cet égard de souligner que l'aire sémantique du mot tuteur ne couvre pas intégralement celle du mot wali. Peut-on traduire wali par tuteur lorsqu'il s'agit d'une personne chargée de veiller sur une femme majeure et saine d'esprit, en sachant que le mot tuteur désigne la personne chargée de veiller uniquement sur un mineur ou une personne majeure inapte, d'administrer ses biens et de le représenter dans les actes juridiques? De toute évidence, non.

Le mot wali est tantôt mis entre guillemets («wali »), tantôt inséré sans guillemets dans le texte. C'est aussi le cas du mot chari'â qui est, lui, tantôt mis entre guillemets, tantôt entre parenthèses. À la fin de l'article 222, on le voit même écrit avec un «a » à la fin : chari'âa.

Cette confusion entre mise entre guillemets et mise entre parenthèses, et ce va-et-vient entre celles-ci et l'insertion directe dans le texte, révèlent les difficultés qu'éprouve le traducteur juridique algérien à traduire certains termes juridiques arabes en langue française. Celui-ci n'opte pas pour une forme de traduction de manière claire et tranchée. Il est tiraillé entre sa volonté de recourir à des mots français susceptibles de rendre les termes arabes avec la crainte de ne pas les rendre ainsi de manière pleine (l'exemple du mot tuteur), et celle de recourir à des transcriptions phonétiques (Idda, Hadana, Sadaq el mithl, etc.) qu'il sait difficilement accessibles, voire carrément étrangères à un lecteur non arabophone, francophone en l'occurrence. 
En effet, les mots El Kbitba, Idda, Tabanni, wali, Hadana, Sadaq el mithl, etc. sont tous des termes très imprégnés de connotations religieuses. Leur citation dans le Coran - pour certains et leur adoption par le droit musulman les a façonnés et revêtus d'une dimension de sacralité à tel point que les rendre dans une autre langue et dans un autre système dont les termes ont été de plus en plus sécularisés à travers le temps devient une tâche assez compliquée, voire impossible à réaliser de manière satisfaisante. S’agit-il en réalité de notions juridiques sans équivalence?

Le traducteur juridique est souvent confronté à un problème de non-équivalence juridique entre les notions se trouvant dans le texte source et les notions de la langue du texte d'arrivée lorsqu'il tente un transfert d'une culture juridique à une autre. Ceci semble se confirmer dans la traduction du Code de la famille algérien qui, beaucoup plus que les autres textes de loi (le Code civil ou le Code pénal par exemple), est très fortement pénétré par le droit musulman 8

Pour contourner cette difficulté majeure, plusieurs solutions sont envisageables. La première consisterait à amener le traducteur à interpréter la notion juridique telle qu'elle se trouve dans la langue de départ puis à lui chercher un mot correspondant dans la langue d'arrivée.

Cette approche est jugée "critiquable et risquée » par Abdel Hadi Maher qui affirme que celle-ci «transforme le traducteur en interprète au sens étymologique du terme, c'est-à-dire une personne chargée non pas de traduire un sens dans une langue vers une autre, mais également d'interpréter dans la langue d'arrivée la notion juridique couverte par le terme déjà employé dans la langue source. » (76). Pour l'auteur, cette approche dévie le traducteur de son rôle principal qui consiste à opérer un transfert linguistique et non pas à faire œuvre de pédagogue. En outre, elle exige du traducteur, en plus des connaissances linguistiques, des connaissances juridiques très appuyées lui permettant d'être en mesure d'expliciter des notions de droit en langue étrangère, faisant ainsi de lui le juriste qu'il n'est pas forcément censé être au départ. D’où le risque qu'il encourt, vu l'ambigüité de certains termes juridiques, de commettre des erreurs qui pourraient s'avérer graves.

Pour lui, la solution la plus appropriée serait la transcription phonétique. Le traducteur doit ainsi se contenter de prendre le terme tel qu'il est en langue source et de le transcrire phonétiquement en langue cible. Il appartiendra par la suite au lecteur lui-même de faire l'effort de cerner la portée exacte du terme dont il s'agit au lieu que l'effort d'interprétation juridique soit fourni par le traducteur lui-même avec tous les risques que cela suppose.

C'est justement la solution qui semble être privilégiée par le traducteur du Code de la famille algérien. Le recours à la transcription phonétique en français de termes arabes est en effet quasi systématique (El khitba, Idda, Tabbani, Hadana, etc.).

Néanmoins, n'est-ce pas céder à la facilité que de recourir systématiquement à cette forme de traduction? Le texte produit par une telle traduction risque d'être complètement ésotérique alors que l'opération traduisante vise à mettre celui-ci à la portée du plus grand nombre, d'autant plus que, contrairement aux autres langues de spécialité, le langage du droit tombe sous le sceau de l'exigence d'accessibilité aux non-initiés. Nul n'est censé ignorer la loi selon l'adage populaire.

S’il est vrai que le traducteur n'est pas censé faire œuvre d'interprète ou de pédagogue en explicitant dans le texte d'arrivée chaque terme juridique appartenant à la culture juridique du texte de départ, il est tout aussi vrai qu'il n'appartient pas au destinataire - de surcroît pas

\footnotetext{
${ }^{8}$ Il faut rappeler que ce Code de la famille a longtemps soulevé l'indignation des défenseurs des droits des femmes et des militantes féministes algériennes qui le jugent rétrograde.
} 
toujours initié - de percer l'ésotérisme de certains termes qui, étant transcrits phonétiquement, lui sont doublement étrangers : sur le plan purement linguistique (différence de langue) et sur le plan culturel (différence de culture juridique).

Une autre solution nous viendrait de la création discursive. Celle-ci étant, selon les termes de Jean Delisle, une formulation qui «n'est pas créée de toutes pièces, elle [l'expression] existe de façon latente dans la langue d'arrivée, mais elle n'est pas associée normalement aux mots du texte de départ et ne figure dans aucun dictionnaire bilingue. » (168). Elle permet ainsi au traducteur de formuler une expression nouvelle à partir d'un terme arabe afin de couvrir la notion juridique inexistante en droit français. C'est la solution choisie par le traducteur juridique algérien lorsqu'il traduit la notion juridique islamique de «Kafala» par l'expression française « recueil légal». On peut lire notamment dans l'article $116:$ :Le recueil légal est l'engagement de prendre bénévolement en charge l'entretien, l'éducation et la protection d'un enfant mineur, au même titre que le ferait un père pour son fils. Il est établi par acte légal.» Étant différent de Tabanni, c'est-à-dire l'adoption proprement dite, qui, elle, est complètement interdite par le droit musulman, Kafala est un concept juridique particulier aux pays musulmans qui s'inspire essentiellement de certains préceptes du Coran se rapportant à la question de la filiation. Transférer un tel terme chargé de connotations religieuses dans une autre langue que l'arabe et dans une culture juridique autre que musulmane revient immanquablement à le séculariser. La création discursive n'enlève rien à cette sécularisation, loin s'en faut !

Il est à cet égard utile de souligner que le statut de la langue arabe demeure très particulier. Il est étroitement lié à l'existence du Coran. L'arabe est perçu par certains comme la langue de la parole divine et elle est, ce faisant, la seule à pouvoir préserver la sacralité des termes tirés du droit musulman dont elle subit fortement l'influence. Toute traduction serait donc synonyme d'une sorte d'altération de cette dimension sacrale dont jouissent des termes énoncés à l'origine en langue arabe dans le texte sacré qu'est le Coran.

Le droit est en effet un phénomène local qui franchit difficilement les frontières. Il est de ce fait très imprégné de la langue du terroir et de la culture qu'elle porte, des us et coutumes et de l'histoire de la communauté sociale qui l'a vu naitre et évoluer. Il remonte aux sources de la civilisation. À la différence des spécialités scientifiques et techniques, le droit, étant ancré dans des communautés humaines distinctes, ne dispose pas de référents opératoires universels. C'est pourquoi les termes du droit sont le plus souvent marqués culturellement. Comme l'a énoncé le juge canadien Pigeon (273), «en traduction juridique, lorsqu'on est en présence de termes spécialisés, la difficulté de la traduction, loin d'être éliminée, comme dans d'autres domaines se trouve amplifiée. C'est qu'il y a une relation étroite entre chaque concept juridique et la langue dans laquelle il a été élaboré ».

Le problème majeur de la traduction juridique apparaît donc, à la base, au niveau terminologique, car en plus de transmettre un message d'une langue à une autre, il faut le transmettre d'un système juridique à un autre, c'est-à-dire d'un système ayant ses propres concepts, élaborés tout au long de son histoire et intimement liés à une tradition juridique déterminée, à un autre système ayant des concepts différents, issus d'une tradition juridique tout aussi différente.

C'est, à l'évidence, à la terminologie appliquée au droit que l'on fait référence lorsque l'on parle de terminologie juridique. Le problème avec cette dernière c'est qu'il s'agit, d'une part, d'une terminologie technique, c'est-à-dire se rapportant à un domaine technique, celui de la science juridique, mais c'est aussi, d'autre part, une terminologie qui se rapporte à une science sociale et 
non pas à une science exacte. Il résulte de cette caractéristique que la traduction d'un texte juridique fera apparaître deux principales sources de difficultés terminologiques.

D’une part, le traducteur devra déceler la frontière, aussi ténue soit-elle, entre ce qui relève de la langue du droit et ce qui relève de la langue courante. C'est surtout la polysémie du vocabulaire juridique qui lui posera ici problème. D'autre part, les termes du droit sont le plus souvent culturellement marqués : c'est toute une culture juridique musulmane qui transparait tout naturellement derrière un terme juridique arabe.

En effet, tout système juridique entretient immanquablement un rapport étroit avec la langue qui l'a vu naître. La langue détermine souvent l'appartenance à tel ou tel autre système juridique, et celui-ci, à son tour, imprime de son sceau la langue qui le véhicule. Ceci n'est évidemment pas sans conséquences. Le fait que le système juridique ait une influence sur la langue du droit implique que chaque concept soit exprimé par un mot particulier dans chaque langue déterminée. Le découpage de la réalité varie d'une langue à une autre et celui de la réalité juridique varie d'un système juridique à un autre. Ceci implique que chaque système juridique opte pour le choix de tel mot au détriment de tel autre pour exprimer et définir un concept juridique particulier.

En outre, le traducteur doit aussi se poser la question de savoir si la version en langue étrangère (la traduction) est élaborée à seule fin informative ou si elle aura les effets juridiques de l'original. Si la finalité de l'opération traduisante est de créer des droits, elle posera plus de problèmes dans les choix à opérer pour le traducteur. Par contre, si la finalité est purement informative, cette difficulté sera moindre. Le degré de difficulté sera donc déterminé, dans une large mesure, par l'usage auquel est prédestinée la traduction.

Officiellement, c'est au texte rédigé en langue arabe qu'appartient la tâche de créer des droits en Algérie. La version en langue française ne serait donc rédigée qu'à titre informatif?

\section{Conclusion}

Des trois solutions dont dispose le traducteur algérien - l'interprétation de la notion juridique dans la langue d'arrivée, la transcription phonétique et la création discursive - laquelle est plus à même de rendre compte des termes juridiques arabes? Il serait une erreur d'opter pour l'une ou l'autre de ces trois solutions envisageables à l'exclusion des autres. Chacune peut s'avérer la mieux appropriée lors de la traduction de tel ou tel autre terme juridique arabe.

En outre, ne serait-il pas vain de chercher à tout prix à rendre la dimension sacrale des termes juridiques arabes tirés du droit musulman quand on sait qu'il n'y a jamais équivalence parfaite en traduction, les mots n'ayant en effet pas la même extension sémantique dans deux langues différentes?

Quelles que soient les langues en présence, le traducteur du texte juridique doit franchir un double obstacle. D’une part un obstacle linguistique dû à la différence des systèmes linguistiques, et d'une autre part un obstacle culturel, tenant à la différence des cultures juridiques.

\footnotetext{
${ }^{9}$ Le secteur de la justice, comme tous les autres champs de la vie sociale en Algérie, a subi la politique d'arabisation. Il est donc logique de penser que la version française est rédigée pour des fins purement informatives. Cependant, les choses sont autrement plus compliquées. Selon certains spécialistes, on serait en face d'un vrai bilinguisme juridique, les versions arabe et française créant toutes deux du droit.
} 
Dans leurs pratiques professionnelles, les traducteurs juridiques parviennent à contourner les difficultés manifestes résultant de l'empreinte culturelle des termes juridiques en recourant à différentes techniques de traduction. Il est donc plus approprié de parler de degré de traduisibilité au lieu d'intraduisibilité absolue des termes et, ainsi, des textes juridiques. 


\section{Bibliographie}

Babadji, Ramdane. «Désarroi bilingue : note sur le bilinguisme juridique en Algérie.» Droit et Société 15, 1990. 207-217.

Delisle, Jean. La Traduction raisonnée : manuel d'initiation à la traduction professionnelle de l'anglais vers le français. Ottawa : Les Presses de l'Université d'Ottawa, 2 e édition, 2003.

Didier, Emmanuel. «La Common Law en français. Étude juridique et linguistique de la common law en français au Canada. » Revue internationale de droit comparé 1, 1991. 7-56.

Gémar, Jean-Claude. Traduire ou l'art d'interpréter : langue, droit et société : élément de jurilinguistique. Sainte-Foy : Presses de l’Université du Québec, 1995.

Koustivitis, Vassilis G. «La traduction juridique : standardisation versus créativité. » Meta 35-1, 1990. 226-229.

Maher, Abdel Hadi. «La juritraductologie et le problème des équivalences des notions juridiques en droit des pays arabes », ILCE $A, 3$ | 2002, [En ligne], mis en ligne le 8 juin 2010. URL: http://ilcea.revues.org/index816.html.

Pigeon, L.P. : « La traduction juridique : l'équivalence fonctionnelle. » Langage du droit et traduction. Essais de jurilinguistique. Ed. J.C. Gémar. Montréal : Linguatech Collection et Conseil de la langue française, 1982. 